\title{
ON A PROBLEM OF MAHLER FOR TRANSCENDENCY OF FUNCTION VALUES
}

\author{
KUMIKO NISHIOKA \\ (Received 28 May 1981; revised 7 January 1982) \\ Communicated by A. J. van der Poorten
}

\begin{abstract}
A transcendence theorem is proved for functions satisfying functional equations of the shape $P\left(z, f(z), f\left(z^{p}\right)\right)=0$, where $P$ is a polynomial and $p \geqslant 2$ is an integer.

1980 Mathematics subject classification (Amer. Math. Soc.): $10 \mathrm{~F} 35$.
\end{abstract}

\section{Introduction}

In a sequence of three papers, K. Mahler $(1929,1930 \mathrm{a}, 1930 \mathrm{~b})$ discussed the arithmetic properties of functions in several complex variables satisfying a certain type of functional equation. Mahler (1969) gave a summary of his earlier work and proposed three problems connected with it. Two of the three problems have been studied by K. K. Kubota, J. H. Loxton, A. J. van der Poorten and D. W. Masser. (An account of the progress up to 1977 is given in Loxton and van der Poorten (1977).) The present investigation is concerned with the remaining problem. Specifically, this problem asks for the transcendency at algebraic points of functions $f(z)$ satisfying algebraic functional equations of the form $P\left(z, f(z), f\left(z^{p}\right)\right)=0$, where $P$ is a polynomial and $p \geqslant 2$ is an integer. The same question can be asked for functions of several complex variables, with an appropriate generalization of the transformation $z \rightarrow z^{p}$. We shall consider only functions of one variable, but we generalize the basic transformation $z \rightarrow z^{p}$ in a different way, as follows.

(c) Copyright Australian Mathematical Society 1982 
Define the transformation $T$ on the complex plane by

$$
T z=t_{p} z^{p}+t_{p+1} z^{p+1}+\cdots+t_{p+N} z^{p+N},
$$

where $t_{p}, \ldots, t_{p+N}$ are algebraic numbers, $p$ and $N$ are integers with $p>1$ and $N \geqslant 0$, and $t_{p} t_{p+N} \neq 0$. We set

$$
U=\max \left\{1,\left|t_{p}\right|+\cdots+\left|t_{p+N}\right|\right\} .
$$

We shall consider functions $f(z)$ satisfying functional equations of the shape

$$
Q_{0}(z, f(z)) f(T z)^{n}+Q_{1}(z, f(z)) f(T z)^{n-1}+\cdots+Q_{n}(z, f(z))=0
$$

where the $Q_{i}(z, u)$ are relatively prime polynomials with algebraic coefficients and $Q_{0}(z, u)$ is not identically zero. Then we can find polynomials $g_{i}(z, u)$ with algebraic coefficients such that

$$
g(z)(\text { say })=\sum_{i=0}^{n} g_{i}(z, u) Q_{i}(z, u)
$$

is independent of $u$ and not identically zero. We set

$$
m=\max _{0 \leqslant i \leqslant n} \operatorname{deg}_{u} Q_{i}(z, u) \text { and } M=\max \{p+N, m\} .
$$

As usual, if $\alpha$ is an algebraic number, we denote by $\alpha$ the maximum of the absolute values of the conjugates of $\alpha$ and by $d(\alpha)$ the least positive integer such that $d(\alpha) \alpha$ is an algebraic integer, and we set $\operatorname{size}(\alpha)=\max \{\log \lceil\alpha, \log d(\alpha)\}$.

We can now state out theorem, using the notation established above.

THEOREM. Let $f(z)=\sum_{h=0}^{\infty} a_{h} z^{h}$ be a power series whose coefficients all lie in a fixed algebraic number field and suppose that $f(z)$ converges in some neighbourhood of the origin, satisfies the functional equation (1.2) and is not an algebraic function. Let $d_{h}$ be the least positive integer such that $d_{h} a_{j}$ is an algebraic integer for $0 \leqslant j \leqslant h$ and suppose that

$$
\log a_{h}, \log d_{h} \leqslant c h^{L} \quad(h \geqslant 1),
$$

for some $c>0$ and $L \geqslant 1$. Let $\alpha$ be an algebraic number with $0<U|\alpha|<1$ such that $f(\alpha)$ converges and $T^{i} \alpha$ and $g\left(T^{i} \alpha\right)$ are non-zero for $i \geqslant 0$. If

$$
M(p+N) n^{2}<p^{2+1 / L},
$$

then $f(\alpha)$ is transcendental.

For the extension of previous work required to deal with algebraic functional equations, it is necessary to invoke a quantitative form of Siegel's lemma in the construction of the auxiliary function given below. This is the reason for the appearance of the hypotheses (1.3). Unfortunately, the theorem cannot be applied to such interesting functions as $u(\log z / 2 \pi i)-z^{-1}$, where $j(w)$ is the modular 
invariant, because (1.4) is not satisfied. The following are some elementary instances of the theorem not covered by previous work.

(1) The function $f_{p n}(z)=\prod_{h=0}^{\infty}\left(1-z^{p^{h}}\right)^{n^{h}}$ satisfies the functional equation $(1-z) f_{p n}\left(z^{p}\right)^{n}=f(z)$. If $0<n<p^{1 / 2}$ and $\alpha$ is an algebraic number with $0<|\alpha|<1$, then the hypotheses of the theorem are satisfied and $f(\alpha)$ is transcendental.

(2) Let $P(z)$ be a polynomial with algebraic coefficients such that $P(0)=1$. The function $F_{p n}(z)=\prod_{h=0}^{\infty} P\left(z^{p^{h}}\right)^{n^{h}}$ satisfies the functional equation $P(z) F_{p n}\left(z^{p}\right)^{n}=F_{p n}(z)$ and we can take $L=1$ in (1.3). If $P(\beta)=0$ for some $\beta$ with $|\beta|<1$, then $F(z)$ is not an algebraic function because it has infinitely many zeros at $\beta, \beta^{1 / p}, \ldots$ inside its circle of convergence $\{z:|z|<1\}$. (Some care is needed here, in view of the example $\prod_{h=0}^{\infty}\left(1+z^{2^{h}}\right)=(1-z)^{-1}$.) Thus, if $0<n$ $<p^{1 / 2}$ and $\alpha$ is an algebraic number such that $0<|\alpha|<1$ and $P\left(\alpha^{p^{i}}\right) \neq 0$ for $i \geqslant 0$, then $F_{p n}(\alpha)$ is transcendental.

(3) Define $T$ as in (1.1) and suppose that $p+N<p^{3 / 2}$. The function $f(z)=$ $\sum_{h=0}^{\infty} T^{h} z$ converges in $\left\{z:|z|<U^{-1}\right\}$ and satisfies the functional equation $f(T z)=f(z)-z$, so $f(z)$ is not an algebraic function. If $\alpha$ is an algebraic number, $0<U|\alpha|<1$ and $T^{i} \alpha \neq 0$ for $i \geqslant 0$, then $f(\alpha)$ is transcendental.

\section{Preliminary lemmas}

The first lemma is elementary, but useful in estimating the size of a root of a polynomial with algebraic coefficients.

Lemma 1. Suppose that $A_{0} \neq 0, A_{1}, \ldots, A_{n}$ are algebraic numbers and $A_{0} \beta^{n}+$ $A_{1} \beta^{n-1}+\cdots+A_{n}=0$. Then

$$
\left|\overrightarrow{A_{0} \beta}<\right| \overrightarrow{A_{0}}+\left|\overrightarrow{A_{1}}+\cdots+\right| A_{n} .
$$

Further, if $A$ is a positive integer such that $A A_{0}, A A_{1}, \ldots, A A_{n}$ are algebraic integers, then so is $A A_{0} \beta$.

The next two lemmas deal with the interates of the transformation $T$ defined in (1.1).

LEMMA 2. Let $\alpha$ be an algebraic number. Then there are a constant $c_{1}$ and a positive integer $D$, independent of $r$, such that $\left|T^{r} \alpha\right| \leqslant c_{1}^{(p+N)^{r}}$ and $D^{(p+N)^{r}} T^{r} \alpha$ is an algebraic integer for all $r \geqslant 0$. 
Proof. We verify by induction that

$$
\left|T^{r} \boldsymbol{\alpha}\right| \leqslant c_{1}^{1+(p+N)+\cdots+(p+N)^{r}}
$$

for a suitable constant $c_{1}$, and that

$$
D^{1+(p+N)+\cdots+(p+N)^{r}} T^{r} \boldsymbol{\alpha}
$$

is an algebraic integer for a suitable positive integer $D$. This strategy is typical of several similar calculations in what follows.

Lemma 3. If $0<U|\alpha|<1$, then $\left|T^{r} \alpha\right| \leqslant(U|\alpha|)^{p^{r}}$ for $r \geqslant 0$ and $\left|T^{r} \alpha\right|<|\alpha|$ for $r \geqslant 1$.

Proof. We verify by induction that

$$
\left|T^{r} \alpha\right| \leqslant U^{1+p+\cdots+p^{r-1}}|\alpha|^{p^{r}} .
$$

In connection with one of the hypotheses of the theorem, it follows from Lemma 3 that, if

$$
0<U|\alpha|<\min \left\{1,\left|t_{p}\right| /\left(\left|t_{p+1}\right|+\cdots+\left|t_{p+N}\right|\right)\right\},
$$

then $T^{r} \alpha \neq 0$ for $r \geqslant 0$. Indeed, if $T^{r} \alpha=0$, then

$$
\begin{aligned}
\left|t_{p}\right| & =\left|t_{p+1}\left(T^{r-1} \alpha\right)+\cdots+t_{p+N}\left(T^{r-1} \alpha\right)^{N}\right| \\
& \leqslant\left(\left|t_{p+1}\right|+\cdots+\left|t_{p+N}\right|\right)(U|\alpha|)^{p^{r-1}} .
\end{aligned}
$$

\section{Proof of the theorem}

Let the power series $f(z)$ and the number $\alpha$ satisfy all the requirements of the theorem and suppose, in addition, that $f(\alpha)$ is algebraic. Under these assumptions, we shall derive a contradiction, thereby proving the theorem. Let $F$ be an algebraic number field containing the coefficients $t_{p}, \ldots, t_{p+N}$ of the transformation polynomial, all the coefficients of the power series $f(z)$, the coefficients of the polynomials $Q_{0}(z, u), \ldots, Q_{n}(z, u)$ appearing in the functional equation, and the number $\alpha$ and $f(\alpha)$. We assume, as we may, that the coefficients of the polynomials $Q_{0}(z, u), \ldots, Q_{n}(z, u)$ are algebraic integers.

Introduce the parameter $w$ by

$$
p^{2 w / L}=M^{-1}(p+N) p^{1 / L} .
$$


From the definition of $M$ and the assumption (1.4), we have $0<w \leqslant 1 / 2$. Further, by the assumption (1.4),

$$
n M p^{w / L}=n(p+N) p^{(1-w) / L}=\left(n^{2} M(p+N) p^{1 / L}\right)^{1 / 2}<p^{1+1 / L} .
$$

Consequently, we can choose numbers $q$ and $\varepsilon$ such that
$1<q<p^{1 / L}, \quad \varepsilon>1$,
$\varepsilon n M q^{w}<p q$
$n(p+N) q^{1-w}<p q$.

In what follows, $c_{2}, c_{3}, \ldots$ denote positive constants depending only on the quantities introduced above.

By Lemma 3, $\left|T^{r} \alpha\right|<|\alpha|$ for $r \geqslant 1$, so all the series $f\left(T^{r} \alpha\right)$ converges and satisfy

$$
Q_{0}\left(T^{r} \alpha, f\left(T^{r} \alpha\right)\right) f\left(T^{r+1} \alpha\right)^{n}+\cdots+Q_{n}\left(T^{r} \alpha, f\left(T^{r} \alpha\right)\right)=0 .
$$

Now, $g\left(T^{r} \alpha\right) \neq 0$ by hypothesis, so at least one of $Q_{0}\left(T^{r} \alpha, f\left(T^{r} \alpha\right)\right)$, $\ldots, Q_{n-1}\left(T^{r} \alpha, f\left(T^{r} \alpha\right)\right)$ is non-zero. We set

$$
j_{r}=\min \left\{j: Q_{j}\left(T^{r} \boldsymbol{\alpha}, f\left(T^{r} \boldsymbol{\alpha}\right)\right) \neq 0\right\}
$$

and define $Y_{r}(r \geqslant 0)$ inductively, as follows:

$$
Y_{0}=1, \quad Y_{r}=Q_{j_{r-1}}\left(T^{r-1} \alpha, f\left(T^{r-1} \alpha\right)\right) Y_{r-1}^{m} \quad(r \geqslant 1) .
$$

Thus $Y_{r} \neq 0$ for all $r \geqslant 0$. The next lemma gives estimates for these quantities.

Lemma 4. For $r \geqslant 1,\left[F\left(f(T \alpha), \ldots, f\left(T^{r} \alpha\right)\right): Q\right] \leqslant c_{2} n^{r}$ and

$$
\operatorname{size}\left(Y_{r}\right), \operatorname{size}\left(Y_{r} f\left(T^{r} \alpha\right)\right) \leqslant c_{3} r M^{r}
$$

Proof. The assertions follow by induction, using (3.2) and Lemma 1 and 2.

The next lemma, involving the construction of the auxiliary function, is the central point of the proof.

LemMA 5. Let $k$ be a positive integer and set $\rho_{1}=2\left[q^{w k}\right]$ and $\rho_{2}=2\left[q^{(1-w) k}\right]$. Then there are $\rho_{1}$ polynomials $P_{j}(z)=\sum_{i=0}^{\rho_{2}-1} b_{j i} z^{i}\left(0 \leqslant j \leqslant \rho_{1}-1\right)$ with degrees at most $\rho_{2}-1$ and whose coefficients are algebraic integers in $F$ with sizes at most $c_{4} k q^{L k}$, such that the function

$$
E_{k}(z)=\sum_{j=0}^{\rho_{1}-1} P_{j}(z) f(z)^{j}=\sum_{h=0}^{\infty} b_{h} z^{h}
$$

is not identically zero, but all the coefficients $b_{h}$ with $h<(1 / 2) \rho_{1} \rho_{2}$ vanish. Further,

$$
\operatorname{size}\left(b_{h}\right)<c_{5} k h^{L} \text { and } \log \left|b_{h}\right|<c_{6}\left(k q^{L k}+h\right) \text {, }
$$

providing $k$ is sufficiently large. 
Proof. Set $f(z)^{j}=\sum_{h=0}^{\infty} a_{j h} z^{h}$ for $j \geqslant 0$. It is easily verified that

$$
\left|a_{j h}\right| \leqslant c_{7}^{j+h^{L}} \text { and }\left|a_{j h}\right| \leqslant c_{8}^{j+h} \quad(j, h \geqslant 0) .
$$

The first estimate follows from the hypothesis (1.3) and the second follows from the assumption that $f(z)$ converges in some neighbourhood of the origin. The polynomials $P_{j}(z)$ have in all $\rho_{1} \rho_{2}$ coefficients $b_{j i}$. We can achieve the property required of the auxiliary function $E_{k}(z)$ by choosing $b_{j i}$ to satisfy the $(1 / 2) \rho_{1} \rho_{2}$ linear equations

$$
\sum a_{j, h-i} b_{j i}=0 \quad\left(0 \leqslant h<(1 / 2) \rho_{1} \rho_{2}\right) .
$$

(The sum is taken over all $i$ and $j$ satisfying $0 \leqslant j \leqslant \rho_{1}-1$ and $0 \leqslant i \leqslant$ $\min \left\{\rho_{2}-1, h\right\}$.) The integer $D=\prod_{r=1}^{\rho_{1}} d_{\left[\rho_{1} \rho_{2} / 2 r\right]}$ will serve as a common denominator for all the $a_{j, h-i}$ appearing in these equations. The hypothesis (1.3) gives

$$
\log D \leqslant c_{9}\left((1 / 2) \rho_{1} \rho_{2}\right)^{L} \log \rho_{1} \leqslant c_{10} k q^{L K},
$$

whence, by the remark at the beginning of the proof,

$$
\operatorname{size}\left(D a_{j, h-i}\right) \leqslant c_{11} k q^{L k} \text {. }
$$

By a standard version of Siegel's lemma, as given, for example, in Lang (1966), page 4 , the equations (3.3) have a non-trivial solution in which the $b_{i j}$ are algebraic integers in $F$ and

$$
\operatorname{size}\left(b_{i j}\right) \leqslant c_{4} k q^{L k} \quad\left(0 \leqslant i \leqslant \rho_{1}-1,0 \leqslant j \leqslant \rho_{2}-1\right) .
$$

Since $f(z)$ is a transcendental function, the function $E_{k}(z)$ so constructed is not identically zero. By the construction of $E_{k}(z)$.

$$
b_{h}=\sum a_{j, h-i} b_{j i},
$$

where the sum is taken over all $i$ and $j$ satisfying $0 \leqslant j \leqslant \rho_{1}-1$ and $0 \leqslant i \leqslant$ $\min \left\{\rho_{2}-1, h\right\}$. In estimating $b_{h}$, we can suppose that $h \geqslant(1 / 2) \rho_{1} \rho_{2}$, since otherwise $b_{h}=0$. Now, if $k$ is sufficiently large, we have $h \geqslant q^{k}$, so

$$
\begin{aligned}
\log \left|b_{h}\right| & \leqslant \log \rho_{1} \rho_{2}+\left(\rho_{1}+h^{L}\right) \log c_{7}+c_{4} k q^{L k} \\
& \leqslant c_{12} k h^{L} .
\end{aligned}
$$

Also, the integers $D_{h}=\prod_{r=1}^{\rho_{1}} d_{[h / r]}$ will serve as a common denominator for all the $a_{j, h-i}$ appearing in (3.4), so

$$
\log d\left(b_{h}\right) \leqslant \log D_{h} \leqslant c_{9} h^{L} \log \rho_{1} \leqslant c_{10} k h^{L} .
$$

This gives the required estimate for the size of $b_{h}$. Finally, again using (3.4),

$$
\log \left|b_{h}\right| \leqslant \log \rho_{1} \rho_{2}+\left(\rho_{1}+h\right) \log c_{8}+c_{4} k q^{L k} \leqslant c_{6}\left(k q^{L k}+h\right) .
$$

This completes the proof of the lemma. 
In the final part of the proof, we seek to exploit the fundamental inequality of transcendency theory: if $\beta$ is a non-zero algebraic number, then

$$
\log |\beta| \geqslant-2[Q(\beta): Q] \operatorname{size}(\beta) .
$$

Let $E_{k}(z)$ be the function constructed in Lemma 5 . Let $H$ be the least integer such that $b_{H} \neq 0$ and let $K$ be the integer such that $q^{K} \leqslant H<q^{K+1}$. For $k$ sufficiently large, we have $H \geqslant(1 / 2) \rho_{1} \rho_{2} \geqslant q^{k}$, so that $K \geqslant k$.

LEMMA 6. If $k$ is sufficiently large, then

$$
\left[Q\left(Y_{K}^{\rho_{1}} E_{k}\left(T^{K} \alpha\right)\right): Q\right] \leqslant c_{2} n^{K}
$$

and

$$
\operatorname{size}\left(Y_{K}^{\rho_{1}} E_{k}\left(T^{K} \alpha\right)\right) \leqslant c_{13}\left(\max \left\{\varepsilon M q^{w},(p+N) q^{1-w}\right\}\right)^{K} .
$$

Proof. The first assertion follows at once from Lemma 4. For the second, we use the representation

$$
Y_{K}^{\rho_{1}} E_{k}\left(T^{K} \alpha\right)=\sum_{j=0}^{\rho_{1}-1} P_{j}\left(T^{K} \alpha\right)\left(Y_{K} f\left(T^{K} \alpha\right)\right)^{j} Y_{K}^{\rho_{1}-j}
$$

From Lemma 2, 4 and the estimate for the size of the coefficients of the polynomials $P_{j}(z)$ in Lemma 5, we find

$$
\operatorname{size}\left(Y_{K}^{\rho_{1}} E_{k}\left(T^{K} \alpha\right)\right) \leqslant \log \rho_{1} \rho_{2}+c_{4} k q^{L k}+c_{14}(p+N)^{K} \rho_{2}+c_{3} K M^{K} \rho_{1} .
$$

This yields the assertion of the lemma, since $q^{L}<p$ from (3.1).

LEMMA 7. If $k$ is sufficiently large, then $Y_{K}^{\rho_{1}} E_{k}\left(T^{K} \alpha\right) \neq 0$ and

$$
\log \left|Y_{K}^{\rho_{1}} E_{k}\left(T^{K} \alpha\right)\right| \leqslant(1 / 2) p^{K} q^{K} \log (U|\alpha|) .
$$

Proof. We can write

$$
E_{k}\left(T^{K} \boldsymbol{\alpha}\right)=b_{H}\left(T^{K} \alpha\right)^{H}\left\{1+\left(b_{H+1} / b_{H}\right) T^{K} \boldsymbol{\alpha}+\left(b_{H+2} / b_{H}\right)\left(T^{K} \boldsymbol{\alpha}\right)^{2}+\cdots\right\} .
$$

From Lemma 3, the estimates in Lemma 5 and the fundamental inequality (3.5) applied to $b_{H}$,

$$
\begin{aligned}
\log \left|\left(b_{H+h} / b_{H}\right)\left(T^{K} \alpha\right)^{h}\right| & \leqslant c_{6}\left(k q^{L k}+H+h\right)+c_{15} k H^{L}+p^{K} h \log (U|\alpha|) \\
& \leqslant c_{16} K q^{L K}+c_{6} h+p^{K} h \log (U|\alpha|) .
\end{aligned}
$$

We can compare the absolute values of the terms of the series

$$
\left(b_{H+1} / b_{H}\right) T^{K} \alpha+\left(b_{H+2} / b_{H}\right)\left(T^{K} \alpha\right)^{2}+\cdots
$$


with those of the geometric progression

$$
e^{c_{16} K q^{L k}}\left\{e^{c_{6}}(U|\alpha|)^{p^{K}}+\left(e^{c_{6}}(U|\alpha|)^{p^{K}}\right)^{2}+\cdots\right\} \text {. }
$$

By hypotheses, $T^{K} \alpha \neq 0$ and $U|\alpha|<1$. Now, as soon as $e^{c_{6}}(U|\alpha|)^{p^{K}}<1 / 2$, the last series is less than $e^{c_{16} K q^{l . K}} \cdot e^{c_{6}}(U|\alpha|)^{p^{K}} \cdot 2$. If $k$ is sufficiently large,

$$
e^{c_{16} K q^{L K}} \cdot e^{c_{6}}(U|\alpha|)^{p^{K}} \cdot 2<1
$$

since $q^{L}<p$. Thus, $E_{k}\left(T^{K} \alpha\right) \neq 0$ and

$$
\log \left|Y_{K}^{\rho_{1}} E_{k}\left(T^{K} \alpha\right)\right| \leqslant c_{3} K M^{K} \rho_{1}+c_{6}\left(k q^{L k}+H\right)+p^{K} H \log (U|\alpha|)+\log 2,
$$

and the lemma follows from the inequalities in (3.1).

To complete the proof of the theorem, we apply the fundamental inequality (3.5) to the number $Y_{K}^{\rho_{1}} E_{k}\left(T^{K} \alpha\right)$. By Lemma 6 and Lemma 7, we obtain

$$
(1 / 2) p^{K} q^{K} \log (U|\alpha|) \geqslant-2 c_{2} n^{K} c_{13}\left(\max \left\{\varepsilon M q^{w},(p+N) q^{1-w}\right\}\right)^{K},
$$

providing $k$ is sufficiently large. Since $\log (U|\alpha|)<0$ and $K \geqslant k$, this contradicts the choice of the parameters in (3.1).

\section{References}

S. Lang (1966), Introduction to transcendental numbers (Addison-Wesley).

J. H. Loxton and A. J. van der Poorten (1977), 'Transcendence and algebraic independence by a method of Mahler,' Transcendence Theory-Advances and Applications, ed. A. Baker and D. W. Masser, Chapter 15, pp. 211-226 (Academic Press).

K. Mahler (1929), 'Arithmetische Eigenschaften der Lösungen einer Klasse von Funktionalgleichungen,' Math. Ann. 101, 342-366.

K. Mahler (1930a), 'Über das Verschwinden von Potenzreihen mehrerer Veränderlichen in speziellen Punktfolgen', Math. Ann. 103, 573-587.

K. Mahler (1930b), 'Arithmetische Eigenschaften einer Klasse transzendental-transzendenter Funktionen,' Math. Z. 32, 545-585.

K. Mahler (1969), 'Remarks on a paper by W. Schwarz,' J. Number Theory 1, 512-521.

Department of Mathematics

Nara Women's University

Kita-Uoya Nishimachi, Nara 630

Japan 